\title{
A novel timing and frequency offset estimation algorithm for filtered OFDM system
}

\author{
Xing-le Feng $^{1 *}$ (D), Meng-jie Wang ${ }^{1}$, Li Chen² ${ }^{2}$ Wen-xia Zhu ${ }^{1}$ and Kun Hua ${ }^{3}$
}

\author{
${ }^{*}$ Correspondence: \\ xlfeng@chd.edu.cn \\ 'Chang'an University, 710064 Xi'an, \\ China \\ Full list of author information is \\ available at the end of the article
}

\begin{abstract}
As a critical technology of $5 \mathrm{G}$ air interface waveform, filtered orthogonal frequency division multiplexing (F-OFDM) not only inherits the technical advantages of OFDM, but also has outstanding advantages in system flexibility and spectrum efficiency. However, as a multi-carrier technology, it is still extremely sensitive to sample timing offset (STO) and carrier frequency offset (CFO). In this letter, an improved Park frequency domain training sequence (FS-Park) is proposed to complete STO and CFO estimation of F-OFDM system. Firstly, a real-value pseudorandom number (PN) sequence is sent to each subcarrier as training sequence in frequency domain, the corresponding time domain training symbol has a conjugate symmetry structure. Secondly, the training symbol is utilized for timing synchronization, then the fractional frequency offset is estimated based on the cyclic prefix in time domain. Finally, the integer frequency offset is estimated in frequency domain based on the auto-correlation of PN sequence. The simulation results illustrate that the FS-Park algorithm not only has a single pulse timing metric curve and great STO estimation accuracy, but also has better performance of CFO estimation than classical Park algorithm and Liang Xiao's method.
\end{abstract}

Keywords: Filtered orthogonal frequency division multiplexing (F-OFDM), Sample timing offset(STO) estimation, Carrier frequency offset (CFO) estimation, Training sequence, Park algorithm

\section{Introduction}

\subsection{Background knowledge}

With the increasing demands of internet of vehicles (IOV) and internet of things (IOT) business, the wireless data traffic explodes [1]. How to further improve transmission efficiency and spectral utilization has become the research focus of $5 \mathrm{G}$. Now, research on $5 \mathrm{G}$ key waveform technology is aimed at solving the above problems, such as filtered orthogonal frequency division multiplexing (F-OFDM) waveform technology [2]. By means of dividing the whole frequency band into several subbands, F-OFDM achieves flexible configuration of system parameters and low spectrum leakage, then improves data transmission efficiency and the spectral utilization simultaneously. For example, adjacent

(C) The Author(s). 2020 Open Access This article is licensed under a Creative Commons Attribution 4.0 International License which permits use, sharing, adaptation, distribution and reproduction in any medium or format, as long as you give appropriate credit to the original author(s) and the source, provide a link to the Creative Commons licence, and indicate if changes were made. The images or other third party material in this article are included in the article's Creative Commons licence, unless indicated otherwise in a credit line to the material. If material is not included in the article's Creative Commons licence and your intended use is not permitted by statutory regulation or exceeds the permitted use, you will need to obtain permission directly from the copyright holder. To view a copy of this licence, visit http://creativecommons.org/licenses/by/4.0/. 
subcarriers of subband 1 can be allocated with large subcarrier spacing for vehicle to vehicle communication, which can weaken the influence of large doppler frequency shift [3], while adjacent subcarriers of subband 2 can be allocated with small subcarrier spacing for IOT communication to improve system capacity. The two subbands work simultaneously to achieve the optimal utilization of system resources. However, both IOV and IOT business transmission are very sensitive to the sample timing offset (STO) and carrier frequency offset (CFO). Especially in the IOV transmission, the high-speed movement of the vehicle leads to a large doppler frequency shift, which results in a larger CFO. If the CFO cannot be effectively eliminated, larger inter carrier interference (ICI) will be produced [4]. Therefore, STO and CFO synchronization technology is an important prerequisite for F-OFDM system to achieve high-quality and high-rate data transmission [5, 6].

OFDM synchronization algorithms are classified into two types: non-pilot aided method and pilot aided method [7]. The most classical algorithm of non-pilot aided method is the maximum likelihood (ML) algorithm based on cyclic prefix $(\mathrm{CP})$ proposed by Beek in [8], but it only has good performance in the additive white Gaussian noise (AWGN) channel in CFO estimation. Then, Landstorm et al. [9] proposed joint STO and CFO estimation by effectively combining CP and pilot, which can estimate frequency offset effectively in multi-path channel. Due to additional training symbols are added, the pilot aided method has good estimation performance in multi-path channel. Although additional overhead will reduce the transmission rate of the source data, the synchronization accuracy can be guaranteed with low computational complexity [10]. In 5G business, especially in IOV business, multi-path and doppler effect have a greater impact on the system, thus, the pilot aided method is studied in this paper.

Classical pilot aided method mainly include SC algorithm [11], Minn algorithm [12], and Park algorithm [13]. In [11], Schmidl proposed a training symbol like the structure of $[A A B]$, where $[A A]$ represents one training symbol, while $[B]$ represents another one. It is called SC algorithm in the following paper. In this algorithm, if the timing is within the range of $\mathrm{CP}$, the product sum of all the corresponding sample points are equal, which will result in a "peak platform" in the timing metric curve, then lead to a large estimation error of the timing position. In order to solve "peak platform," Minn proposed a training symbol like the structure of $[A A-A-A]$, it can eliminate platform phenomenon by means of introducing negative sign into training symbols [12]. However, the main peak of the timing metric curve is not sharp, and there are several side peaks with significant amplitudes, which leads to a reduction in the precision of timing synchronization. In terms of the problem that the peak value of Minn timing metric curve is not sharp enough, the training symbol like the structure of $\left[A B A^{*} B^{*}\right]$ is designed by B. Park et al. [13]. By analyzing the Park time domain training symbol structure $\left[A B A^{*} B^{*}\right]$ and the timing metric function, its timing metric curve is sharp enough, but the method for generating the time domain structure proposed in [13] cannot obtain the ideal structure $\left[A B A^{*} B^{*}\right.$ ], and the proposed method makes the actual Park timing metric curve still has large side peak. Furthermore, it only can estimate the FFO, which can not have good performance on large CFO.

For F-OFDM system, each subband is equivalent to an independent OFDM modulation. It is necessary to estimate STO and CFO of each subband separately. Aiming at the problem of Park algorithm, an improved STO and CFO estimation is proposed by Liang Xiao in [14]. Although a better timing accuracy can be obtained, the spectrum efficiency 
decreases because three OFDM symbols are employed. Moreover, the estimation range of FFO is too small to complete the whole estimation of FFO, which will affect the subsequent IFO estimation. Thus, it is still necessary to study the synchronization problem of F-OFDM system.

\subsection{Contributions}

In this paper, the synchronization problem of F-OFDM system is mainly studied. The main contributions of the paper are summarized as follows:

1. The influence of STO and CFO on the F-OFDM system is studied and analyzed by mathematical formula. The main reason is there have been many research on the influence of OFDM system synchronization in existing reference, but the analysis of F-OFDM system has not been done yet. The analysis results are given in the form of formulas. It is found from the analysis that, compared with the OFDM system, the STO may cause ISI between subbands in the F-OFDM system, resulting in greater interference of the system, which indicates that timing and frequency synchronization of the F-OFDM system is of great significance.

2. This paper studies and points out the problems of the classical Park algorithm, that is, the difference between the ideal Park algorithm and the actual Park algorithm is analyzed from the theoretical perspective.

3. In view of the high requirement for synchronization in F-OFDM system and a series of disadvantages in the existing synchronization algorithm, a pilot aided synchronization algorithm is proposed in this paper. It estimates STO and CFO of each subband separately, the estimated process is designed as follows. Firstly, a real-value pseudorandom numbers $(\mathrm{PN})$ sequence with samples is sent as training sequence in the frequency domain, whose length is one OFDM symbol. Then, the training symbol with conjugate symmetry structure likes $[A A]$ is obtained in the time domain. When this structure is utilized to estimate the STO, the timing metric has only one non-zero value, thus the pulse timing metric curve can be obtained. Finally, only one training sequence is used to estimate $\mathrm{CFO}$ in the frequency domain. As a result, it realizes the more efficient utilization of spectrum resources than LiangXiao's algorithm, and the CFO estimation range of the improved algorithm can be theoretically expanded to $-\frac{N}{2} \sim \frac{N}{2}$, larger than the estimation range of the classical Park algorithm and the Liang Xiao's method.

The remainder of this paper is organized as follows. The system model is presented in Section 2. The influence of STO and CFO on the F-OFDM system is studied and analyzed in Section 3. The problems of the classical Park algorithm is given in Section 4. Afterwards, a pilot aided synchronization estimation algorithm is proposed in Section 5, which is called FS-Park method. In the Section 6, several experimental results and analyses are presented, which are given to verify the significance of this study. Finally, the conclusion is given in Section 7.

\section{F-OFDM system model description}

The F-OFDM system is optimized on the basis of the OFDM system, its basic modulation method still is OFDM modulation. In this system, it divides the entire frequency band into multiple subbands as required, and then suppresses spectrum leakage by filtering each 
subband and configuring protection subcarriers according to the type of data transmitted by different subbands. Figure 1 is a block diagram of the F-OFDM system.

As can be seen from Fig. 1, the F-OFDM system divides the entire bandwidth into multiple subbands, and each subband can be independently configured with parameters, such as subcarrier spacing, fast Fourier transform (FFT) points, and CP length. Taking an example of subband $l$, the system allocates user $l$ 's data to subband $l$ and configures parameters for it. Then, the data are operated by encoding, subcarrier mapping, inverse fast Fourier transform (IFFT), and add CP in turn.

After $N$ points IFFT and adding $N_{g}$ point as CP , the following results are obtained.

$$
s(n)=\sum_{i=0}^{P-1} s_{i}\left(n-i\left(N+N_{g}\right)\right),
$$

where

$$
s_{i}(n)=\sum_{k=m^{\prime}}^{m^{\prime}+M-1} x_{i}(k) e^{j 2 \pi k n / N},
$$

$x_{i}(k)$ is the data symbol on subcarrier $k$ of OFDM symbol $i, s_{i}(n)$ is OFDM symbol $i, P$ denotes the number of the OFDM symbols, $\left\{m^{\prime}, m^{\prime}+1, \ldots, m^{\prime}+M-1\right\}$ represents the assigned subcarrier range, and $M$ denotes the number of valid subcarriers.

It can be seen from Fig. 1 that, before passing through the filter, the transmitted data of each subband have respectively completed the OFDM modulation. The F-OFDM signal is then obtained by passing the signal $s_{l}(n)$ through an appropriately designed spectrum shaping filter, i.e.,

$$
y_{l}(n)=s_{l}(n) * f_{l}(n),
$$

where $s_{l}(n)$ denotes the OFDM data symbol of the subband $l$, which includes $P$ OFDM symbols. $f_{l}(n)$ is filter impulse response of subband $l$.

The filtered data are superimposed in the time domain. In order to realize the superposition of each subband, it is necessary to maintain the same sampling rate of each subband. That is, the corresponding FFT points are used for IFFT operation, and finally the superimposed time domain data are transmitted through the channel.

$$
y(n)=\sum_{l=0}^{L-1} y_{l}(n),
$$

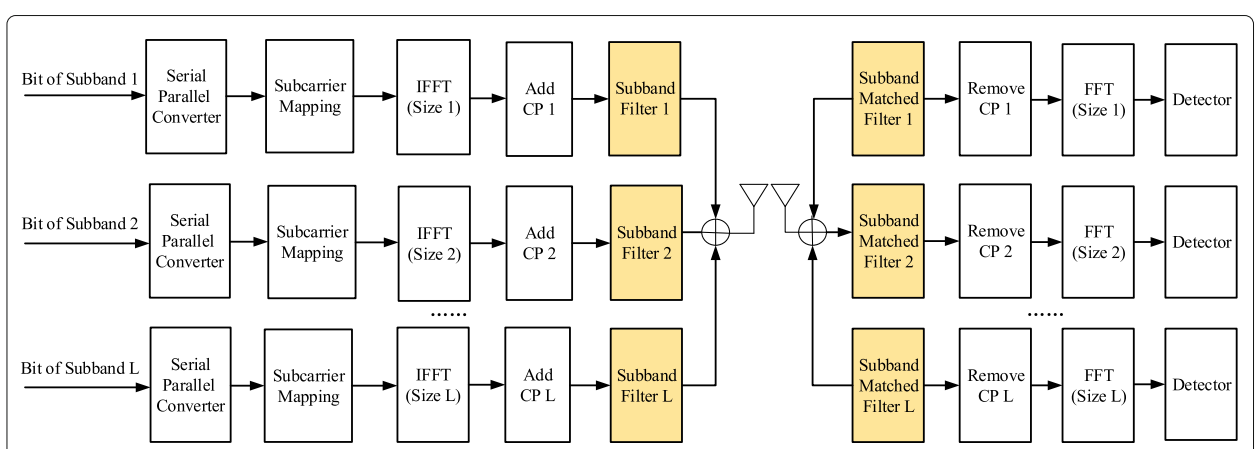

Fig. 1 Block diagram of F-OFDM system 


$$
\begin{aligned}
r(n) & =y(n) * h(n)+w(n) \\
& =\left[\sum_{l=0}^{L-1} y_{l}(n) * h(n)\right]+w(n) \\
& =\left[\sum_{l=0}^{L-1}\left(s_{l}(n) * f_{l}(n) * h(n)\right)\right]+w(n) .
\end{aligned}
$$

In Eqs. (4) and (5), $L$ is the number of subbands. $h(n)$ represents the fading channel impulse response, and $w(n)$ is the AWGN. It should be noted that $h(n)$ has different influences on different subbands, because different subbands can assign different subcarrier spacing. The larger the subcarrier spacing is, the smaller the influence of $h(n)$ on it will be.

The received signal is passed through the filter $f_{l}^{\star}(-n)$, which is matched to the filter used at subband $l$, i.e.,

$$
\begin{aligned}
r_{l}(n) & =r(n) * f_{l}^{\star}(-n) \\
& =\left[\sum_{l=0}^{L-1} s_{l}(n) * f_{l}(n) * h(n)\right] * f_{l}^{\star}(-n) \\
& +w(n) * f_{l}^{\star}(-n) .
\end{aligned}
$$

\section{Influence of synchronization offset on F-OFDM system}

\subsection{Influence of STO on F-OFDM system}

Symbol timing synchronization is applied to correctly ascertain the starting position of the OFDM symbol in each subband, that is, the position of the FFT window. If the starting position is estimated incorrectly, both amplitude and phase of the received signal will be distorted, which may result in the inter symbol interference and deterioration of system performance. A subband symbol timing diagram of F-OFDM system is shown in Fig. 2, and the most accurate symbol timing position should be the first sample point after $\mathrm{CP}$ (case 1). Both timing lead (case 2 and 3) and lag (case 4) will have unfavorable influence on the subsequent demodulation. Taking the subband 1 as an example, the analysis is as follows.

1) When the timing is within the $\mathrm{CP}$ of the current symbol $i$. It means the timing is preceded by $d$ sample value (case 2), in which $d$ represents the number of advanced sampling

\begin{tabular}{|l|l|l|l|}
\hline & & & \\
\hline
\end{tabular}


points. The received values of sample point are given as:

$$
\begin{aligned}
r_{i}(n) & =\frac{1}{N}\left(f_{1}(n) \sum_{k=0}^{N-1} x_{1, i}(k)\right. \\
& \left.+f_{2}(n) \sum_{k=0}^{N-1} x_{2, i}(k)\right) e^{j 2 \pi k(n+d) / N}, \\
n & =0,1, \ldots, N-1
\end{aligned}
$$

where $x_{1, i}(k)$ is the transmitted data on subcarrier $k$ of OFDM symbol $i$ of subband 1 ; $x_{2, i}(k)$ is the transmitted data on subcarrier $k$ of OFDM symbol $i$ of subband 2. $f_{1}(n)$ and $f_{2}(n)$ are filter impulse response of subband 1 and subband 2 , respectively.

$$
\begin{aligned}
s_{1, i}(n) & =g_{1}(n) r_{i}(n) \\
& =\frac{1}{N} \sum_{t=0}^{N_{f}-1} g_{1}(t)\left(f_{1}(n) \sum_{k=0}^{N-1} x_{1, i}(k)+f_{2}(n) \sum_{k=0}^{N-1} x_{2, i}(k)\right) e^{j 2 \pi k(n+d) / N} \\
& =\frac{1}{N}\left(\sum_{t=0}^{N_{f}-1} f_{1}^{*}\left(N_{f}-t\right) f_{1}(t) \sum_{k=0}^{N-1} x_{1, i}(k)+\sum_{t=0}^{N_{f}-1} f_{1}^{*}\left(N_{f}-t\right) f_{2}(t) \sum_{k=0}^{N-1} x_{2, i}(k)\right) e^{j 2 \pi k(n+d) / N} \\
& =\frac{1}{N} \sum_{t=0}^{N_{f}-1}\left|f_{1}(t)\right|^{2} \sum_{k=0}^{N-1} x_{1, i}(k) e^{j 2 \pi k(n+d) / N} \\
& =\frac{1}{N} \sum_{k=0}^{N-1} x_{1, i}(k) e^{j 2 \pi k(n+d) / N} .
\end{aligned}
$$

Through matched filter at the receiver side, without considering inter-subband interference, the subband 1 signal is obtained as Eq. (8), where, $N_{f}$ denotes the length of the filter, $g_{1}(n)$ is the matched filter impulse response at the receiver side of subband 1 , $\sum_{t=0}^{N_{f}-1}\left|f_{1}(t)^{2}\right|=1, \sum_{t=0}^{N_{f}-1} f_{1}\left(N_{f}-t\right) f_{2}(t)=0$.

After the FFT demodulation in $N$ sampling points, the following result is obtained.

$$
\begin{aligned}
y_{1, i}(k) & =\sum_{n=0}^{N-1} s_{1, i}(n) e^{-j 2 \pi n k / N} \\
& =\frac{1}{N} \sum_{n=0}^{N-1} \sum_{k=0}^{N-1} x_{1, i}(k) e^{j 2 \pi k(n+d) / N} e^{-j 2 \pi n k / N} \\
& =x_{1, i}(k) e^{j 2 \pi k d / N} .
\end{aligned}
$$

It shows that a phase rotation of $e^{j 2 \pi k d / N}$ will be introduced to all the subcarriers of the demodulated symbol $i$ of subband 1, but there is not relative shift between the adjacent subcarriers, which does not cause ICI and ISI. 
2) When the timing is within the previous symbol $i-1$ (case 3 ), the received sample point values are given as:

$$
\begin{aligned}
r_{i}(n) & =\frac{1}{N}\left[f _ { 1 } ( n ) \left(\sum_{k=N-d+N_{g}}^{N-1} x_{1, i-1}(k) e^{j 2 \pi k\left(n-N_{g}+d\right) / N}\right.\right. \\
& \left.+\sum_{k=0}^{N-1} x_{1, i}(k) e^{j 2 \pi k(n+d) / N}-\sum_{k=N-d}^{N-N_{g}-1} x_{1, i}(k) e^{j 2 \pi k(n+d) / N}\right) \\
& +f_{2}(n)\left(\sum_{k=N-d+N_{g}}^{N-1} x_{2, i-1}(k) e^{j 2 \pi k\left(n-N_{g}+d\right) / N}\right) \\
& \left.\left.+\sum_{k=0}^{N-1} x_{2, i}(k) e^{j 2 \pi k(n+d) / N}-\sum_{k=N-d}^{N-N_{g}-1} x_{2, i}(k) e^{j 2 \pi k(n+d) / N}\right)\right] . \\
n= & 0,1, \ldots, N-1
\end{aligned}
$$

Through matched filter at the receiver side, without considering inter-subband interference, the subband 1 signal is obtained as Eq. (11).

$$
\begin{aligned}
s_{1, i}(n) & =g_{1}(n) r_{i}(n) \\
& =\frac{1}{N} \sum_{t=0}^{N_{f}-1}\left|f_{1}(t)\right|^{2}\left(\sum_{k=N-d+N_{g}}^{N-1} x_{1, i-1}(k) e^{j 2 \pi k\left(n-N_{g}+d\right) / N}\right. \\
& \left.+\sum_{k=0}^{N-1} x_{1, i}(k) e^{j 2 \pi k(n+d) / N}-\sum_{k=N-d}^{N-N_{g}-1} x_{1, i}(k) e^{j 2 \pi k(n+d) / N}\right) \\
& =\frac{1}{N}\left(\sum_{k=N-d+N_{g}}^{N-1} x_{1, i-1}(k) e^{j 2 \pi k\left(n-N_{g}+d\right) / N}\right) \\
& \left.+\sum_{k=0}^{N-1} x_{1, i}(k) e^{j 2 \pi k(n+d) / N}-\sum_{k=N-d}^{N-N_{g}-1} x_{1, i}(k) e^{j 2 \pi k(n+d) / N}\right) . \\
n= & 0,1, \ldots, N-1
\end{aligned}
$$

After the FFT operation in $N$ sampling points, the following result is obtained.

$$
\begin{aligned}
y_{1, i}(k) & =\sum_{n=0}^{N-1} s_{1, i}(n) e^{-j 2 \pi n k / N} \\
& =\frac{1}{N}\left[\sum _ { n = 0 } ^ { N - 1 } \left(\sum_{k=N-d+N_{g}}^{N-1} x_{1, i-1}(k) e^{j 2 \pi k\left(n-N_{g}+d\right) / N}\right.\right. \\
& +\sum_{k=0}^{N-1} x_{1, i}(k) e^{j 2 \pi(n+d) / N} \\
& \left.\left.-\sum_{k=N-d}^{N-N_{g}-1} x_{1, i}(k) e^{j 2 \pi k(n+d) / N}\right)\right] e^{-j 2 \pi n k / N} \\
= & x_{1, i}(k) e^{j 2 \pi k d / N}+x_{1, i-1}(k) e^{j 2 \pi k\left(d-N_{g}\right) / N} \\
- & x_{1, i}\left(k-N_{g}\right) e^{j 2 \pi\left(k-N_{g}\right) d / N} .
\end{aligned}
$$


It can be seen from the Eq. (12) that a phase rotation of $e^{j 2 \pi k d / N}$ will be introduced to all the subcarriers of the demodulated symbol $i$ of subband 1. Meanwhile, the symbol is affected by the previous symbol $i-1$ and the current symbol $i$ and thus leads to ICI and ISI. In the F-OFDM system, it is possible to produce ISI between subbands, which makes the interference influence greater.

3) When the timing delays $d$ sample points (case 4), the received sample point values are given as:

$$
\begin{aligned}
r_{i}(n)= & \frac{1}{N}\left[f _ { 1 } ( n ) \left(\sum_{k=0}^{N-1} x_{1, i}(k) e^{j 2 \pi k(n-d) / N}\right.\right. \\
+ & \sum_{k=0}^{d-1} x_{1, i+1}\left(N+k-N_{g}\right) e^{j 2 \pi k(n-d) / N} \\
& \left.-\sum_{k=0}^{d-1} x_{1, i}(k) e^{j 2 \pi k(n-d) / N}\right) \\
+ & f_{2}(n)\left(\sum_{k=0}^{N-1} x_{2, i}(k) e^{j 2 \pi k(n-d) / N}\right. \\
+ & \sum_{k=0}^{d-1} x_{2, i+1}\left(N+k-N_{g}\right) e^{j 2 \pi k(n-d) / N} \\
& \left.\left.-\sum_{k=0}^{d-1} x_{2, i}(k) e^{j 2 \pi k(n-d) / N}\right)\right] . \\
n= & 0,1, \ldots, N-1
\end{aligned}
$$

Through matched filter at the receiver side, without considering inter-subband interference, the subband 1 signal is obtained as follows:

$$
\begin{aligned}
s_{1, i}(n)= & g_{1}(n) r_{i}(n) \\
= & \frac{1}{N} \sum_{t=1}^{N_{f}-1}\left|f_{1}(t)\right|^{2}\left(\sum_{k=0}^{N-1} x_{1, i}(k) e^{j 2 \pi k(n-d) / N}\right. \\
+ & \sum_{k=0}^{d-1} x_{1, i+1}\left(N+k-N_{g}\right) e^{j 2 \pi k(n-d) / N} \\
& \left.-\sum_{k=0}^{d-1} x_{1, i}(k) e^{j 2 \pi k(n-d) / N}\right) \\
= & \frac{1}{N}\left(\sum_{k=0}^{N-1} x_{1, i}(k) e^{j 2 \pi k(n-d) / N}\right. \\
& +\sum_{k=0}^{d-1} x_{1, i+1}\left(N+k-N_{g}\right) e^{j 2 \pi k(n-d) / N} \\
& \left.\quad \sum_{k=0}^{d-1} x_{1, i}(k) e^{j 2 \pi k(n-d) / N}\right) .
\end{aligned}
$$


After the FFT operation in $N$ sampling points, the following result is obtained.

$$
\begin{aligned}
y_{1, i}(k) & =\sum_{n=0}^{N-1} s_{1, i}(n) e^{-j 2 \pi n k / N} \\
& =\frac{1}{N} \sum_{n=0}^{N-1}\left(\sum_{k=0}^{N-1} x_{1, i}(k) e^{j 2 \pi k(n-d) / N}\right. \\
& +\sum_{k=0}^{d-1} x_{1, i+1}\left(N+k-N_{g}\right) e^{j 2 \pi k(n-d) / N} \\
& \left.-\sum_{k=0}^{d-1} x_{1, i}(k) e^{j 2 \pi k(n-d) / N}\right) e^{-j 2 \pi n k / N} \\
& =x_{1, i}(k) e^{-j 2 \pi k d / N}+\left(x_{1, i+1}\left(N+k-N_{g}\right)\right. \\
& \left.-x_{1, i}(k)\right) e^{-j 2 \pi k d / N} .
\end{aligned}
$$

Equation (15) shows that a phase rotation of $e^{-j 2 \pi k d / N}$ will be introduced to all the subcarriers of the demodulated symbol $i$ of subband 1 . Meanwhile, the symbol is affected by the symbol $i+1$ and the current symbol $i$ and thus results in ICI and ISI. In the F-OFDM system, it is possible to produce ISI between subbands, which makes the interference influence greater.

\subsection{Influence of CFO on F-OFDM system}

The CFO is usually a random number. In order to simplify analysis and estimation, the normalized frequency offset $\varepsilon$ is ordinarily obtained through dividing it by a subcarrier spacing $\Delta f$, which is divided into IFO $\varepsilon_{I}$ and FFO $\varepsilon_{f}$. Note that $\varepsilon_{I}$ only causes integer periodic shift of subcarriers and does not produce ICI that affects the orthogonality of subcarrier, but $\varepsilon_{f}$ produces ICI. The effects of IFO and FFO on F-OFDM system are analyzed by means of mathematics, respectively.

Take subband 1 as an example, assuming that the timing is accurate and only CFO is existed in the channel, the data influenced by the CFO can be given as:

$$
\begin{aligned}
r(n) & =\frac{1}{N}\left(f_{1}(n) \sum_{k=0}^{N-1} x_{1}(k) e^{j 2 \pi(k+\varepsilon) n / N}\right. \\
& \left.+f_{2}(n) \sum_{k=0}^{N-1} x_{2}(k) e^{j 2 \pi(k+\varepsilon) n / N}\right) .
\end{aligned}
$$

Through matched filter at the receiver side, the subband 1 signal is obtained as follows:

$$
\begin{aligned}
s_{1}(n) & =g_{1}(n) r(n) \\
& =\frac{1}{N} \sum_{t=1}^{N_{f}}\left|f_{1}(t)\right|^{2} \sum_{k=0}^{N-1} x_{1}(k) e^{j 2 \pi(k+\varepsilon) n / N} \\
& =\frac{1}{N} \sum_{k=0}^{N-1} x_{1}(k) e^{j 2 \pi(k+\varepsilon) n / N} .
\end{aligned}
$$


After the FFT operation for $s_{1}(n)$, the demodulation data of subband $1 y_{1}(k)$ will be:

$$
\begin{aligned}
y_{1}(k) & =\sum_{n=0}^{N-1} s_{1}(n) e^{-j 2 \pi k n / N} \\
& =\frac{1}{N} \sum_{n=0}^{N-1}\left(\sum_{i=0}^{N-1} x_{1}(i) e^{j 2 \pi(i+\varepsilon) n / N}\right) e^{-j 2 \pi k n / N} \\
& =\frac{1}{N} \sum_{n=0}^{N-1} \sum_{i=0}^{N-1} x_{1}(i) e^{j 2 \pi(i+\varepsilon-k) n / N}
\end{aligned}
$$

where, $x_{1}(k)$ represents the transmitted data of subband $1, x_{2}(k)$ represents the transmitted data of subband 2.

If there is only IFO, that means $\varepsilon$ is an integer, the Eq. (18) can be redrafted as:

$$
\begin{aligned}
y_{1}(k) & =\sum_{\substack{n=0 \\
i=k-\varepsilon}}^{N-1} x_{1}(k-\varepsilon) \\
& +\sum_{n=0}^{N-1} \sum_{\substack{i=0 \\
i \neq k-\varepsilon}}^{N-1} x_{1}(i) e^{j 2 \pi(i+\varepsilon-k) n / N} \\
& =\sum_{\substack{n=0 \\
i=k-\varepsilon}}^{N-1} x_{1}(k-\varepsilon) .
\end{aligned}
$$

It can be seen from the Eq. (19) that the $\varepsilon_{I}$ does not destroy the orthogonality between the subcarriers, and thus it does not cause ICI. It only causes cyclic shift of the received signal in the frequency domain.

If there is only FFO, that means $\varepsilon$ is a fraction, the Eq. (18) can be redrafted as:

$$
\begin{aligned}
y_{1}(k) & =\sum_{\substack{n=0 \\
i=k}}^{N-1} x_{1}(k) e^{j 2 \pi \varepsilon n / N} \\
& +\sum_{n=0}^{N-1} \sum_{\substack{i=0 \\
i \neq k}}^{N-1} x_{1}(i) e^{j 2 \pi(i+\varepsilon-k) n / N} .
\end{aligned}
$$

From the Eq. (20), it turns out that when there is $\varepsilon_{f}$, the demodulation data on the subcarrier $k$ will suffer not only phase rotation but also ICI, which corresponds to the first and the second parts on the right side of Eq. (20), respectively.

\section{The problem of Park algorithm}

The ideal training symbol structure proposed by Park is $\left[A B A^{*} B^{*}\right]$ [13], which is shown in Fig. 3. Specifically, $[A]$ represents the combination of $[a b c d]$, $[B]$ corresponding to $\left[\begin{array}{lll}d & b & a\end{array}\right]$, and $(\bullet)^{*}$ is the conjugate operation. The calculation rule of the timing metric of Park algorithm is presented in [13].

Park pointed out that the time domain structure like $\left[A B A^{*} B^{*}\right]$ can be achieved in the following cases. A real-valued PN sequence with $N / 2$ length is transmitted on the even subcarriers in frequency domain, while odd subcarriers send zeros. However, this argument has simply been proven wrong by the following demonstration. 


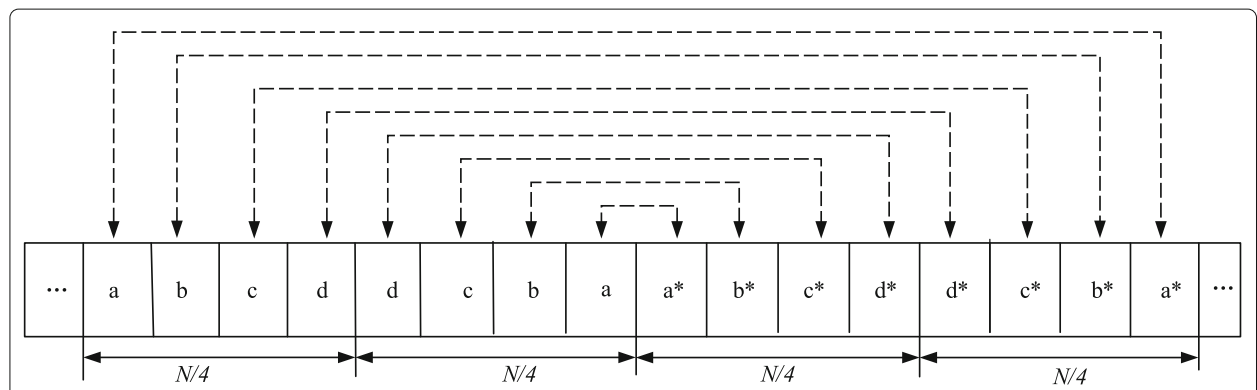

Fig. 3 Ideal time domain symbol structure of Park algorithm

It is well known that a real-valued frequency sequence $x(k)$ with $N$ length will be transformed into $x(n)$ after IFFT. The following equation is true.

$$
x(n)=x^{*}(N-n) .
$$

In addition, if a real-valued $\mathrm{PN}$ sequence of length $N / 2$ is transmitted on the even subcarriers, while zeros are padded on the odd subcarriers, the time domain structure like $[A A]$ can be achieved, in which the length of $[A]$ is $N / 2$. Based on the above properties, the actual structure of the time domain symbol proposed by Park should be the pattern shown in Fig. 4, instead of that in Fig. 3.

Comparing the structures shown in Figs. 3 and 4, it can be noticed that the actual structure obtained by Park algorithm is quite different from the ideal structure $\left[A B A^{*} B^{*}\right.$ ]. The following is a summary of the Park algorithm.

1. Actually, the ideal time domain structure $\left[A B A^{*} B^{*}\right]$ of Park algorithm can not be obtained by the method described in [13]. It can not be generated directly by using the IFFT operation of training sequence in the frequency domain.

2. There is not conjugate symmetric symbol for the first sampling point in the actual structure (that is the first $a$ in Fig. 4), which is different from the ideal structure.

3. The correlation timing metric function proposed by [13] is applicable to the actual time domain symbol structure, whose timing metric curve still has large side peaks. When timing point lies in the $(N / 4+1)$ th point of actual structure, which is just the position of the first $r$ in Fig. 4, due to the existence of $\mathrm{CP}$ and the known feature of the symbol structure, there will be more than $N / 2$ pairs of conjugate pairs in total. In addition, when timing point shift to the $(3 N / 4+1)$ th point, that is the second $r$ in Fig. 4 , there are still $N / 4$ pairs of conjugate pairs. Therefore, there are side peaks at these locations. Undoubtedly, these side peaks will affect the accuracy of timing, which can be verified by Fig. 9c in Section 6.

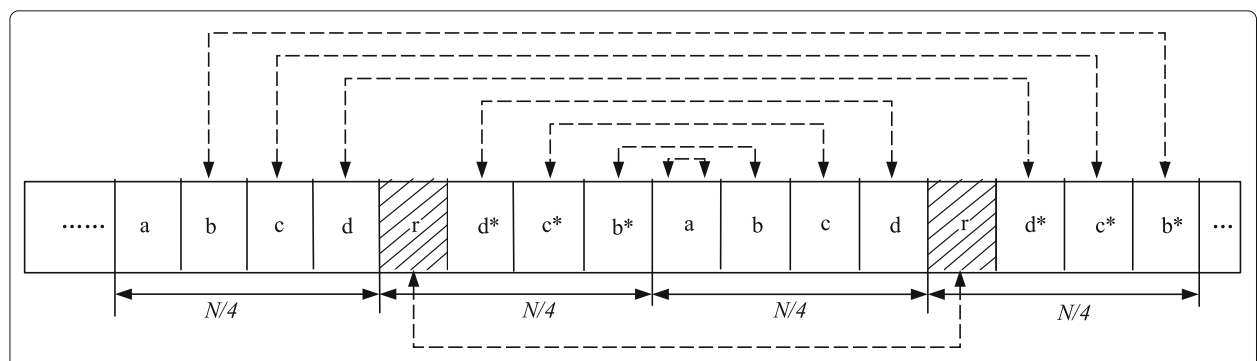

Fig. 4 Actual time domain symbol structure of Park algorithm 
4. By analyzing the ideal training symbol structure $\left[A B A^{*} B^{*}\right]$ of Park algorithm, its correlation timing metric function can be expressed by (22) and (23).

$$
M(s)=\frac{\left|P(s)^{2}\right|}{(R(s))^{2}},
$$

where

$$
\begin{aligned}
& P(s)=\sum_{k=0}^{N / 2-1} r(s+k) r(s-k-1) \\
& R(s)=\sum_{k=0}^{N / 2-1}|r(s+k)|^{2} .
\end{aligned}
$$

It should be noted that the $a, b, c$, etc in Figs. 3 and 4 is just a symbol, without actual meaning.

\section{Synchronization estimation of FS-Park algorithm}

\subsection{STO estimation of FS-Park algorithm}

In order to resolve above problems of Park algorithm, a novel training sequence is proposed in this paper. The training sequence is generated by transmitting a real-valued PN sequence of length $N$ in frequency domain subcarriers, and then the time domain training symbol structure can be obtained by IFFT of $N$ points, which is revealed in Fig. 5 .

As mentioned above, based on the time domain structure shown in Fig. 5, the novel timing metric is defined as:

$$
M(s)=\frac{\left|P(s)^{2}\right|}{(R(s))^{2}},
$$

where

$$
P(s)=\sum_{k=0}^{N / 2-1} r(s-k) r(s+k),
$$

and

$$
R(s)=\sum_{k=0}^{N / 2-1}|r(s+k)|^{2} .
$$

Figure 5 shows that only at the correct point of timing (position $r$ ), $P(s)$ has $N / 2$ conjugate symmetric product pairs; otherwise, the number of conjugate symmetric product

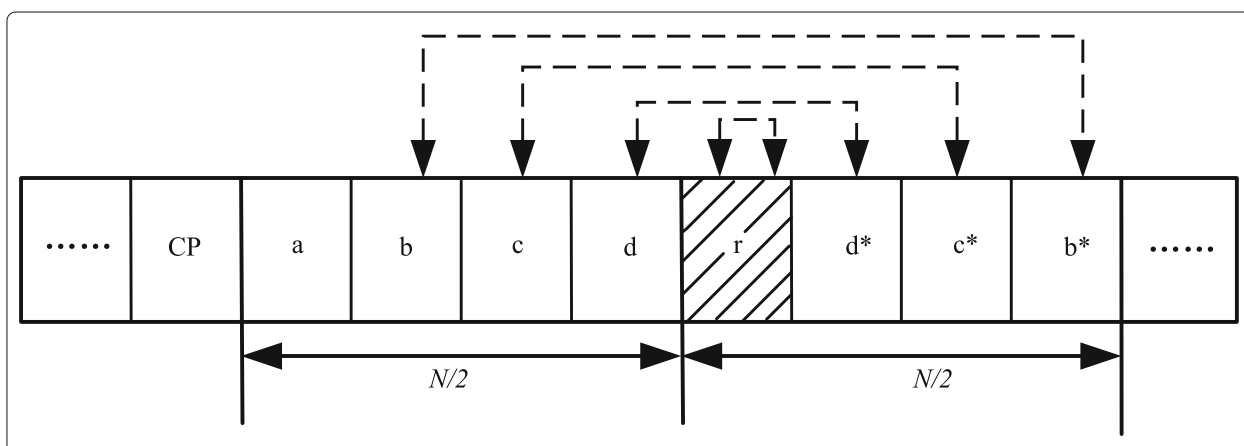

Fig. 5 Time domain symbol structure of FS-Park algorithm 
pairs is zero. So, the correct timing point position can be given as:

$$
\hat{s}=\underset{s}{\arg \max }(M(s))-\frac{N}{2} \text {. }
$$

The timing metric of FS-Park algorithm only generates one non-zero value at the correct point of timing (position $r$ ), and the rest position values are all zero. The timing metric of Park algorithm generates four non-zero values in the whole estimated range, which results in the curve of its timing measurement function having multiple side peaks, while the FS-Park algorithm has no side peak.

\subsection{CFO estimation of FS-Park algorithm}

In general, CFO of OFDM has its fractional and integer parts [15], that is, the total CFO is $\varepsilon=\varepsilon_{f}+\varepsilon_{I}$. Park algorithm only estimates the former part, thus its estimation range is within one subcarrier spacing. In this proposed algorithm, CFO estimation is accomplished by two steps. Firstly, the CP of the symbol is used to estimate $\varepsilon_{f}$ in the time domain, which is based on the phase difference between the original copy of $\mathrm{CP}$ and its duplication. Then, $\varepsilon_{I}$ is estimated by means of the auto-correlation of the training sequence in frequency domain. Since a F-OFDM subband is equivalent to an OFDM system, according to the design rule of OFDM system, CP is an identical copy of the last $N_{g}$ samples of each OFDM symbol, which is appended in front of the symbol. Ideally, if the system has no CFO, the values at the receiver side should be the same. While if the system has FFO of $\varepsilon_{f}$ within one subcarrier spacing, as shown in Fig. 6, supposing the frequency domain signal is sent as $x(n)$, then the received signal in multi-path channel is given as:

$$
\begin{aligned}
r(n)=e^{j 2 \pi \varepsilon_{f} n / N} \sum_{u=0}^{U-1} h_{u} x\left(n-\alpha_{u}\right)+w(n), \\
r(n+N)=e^{j 2 \pi \varepsilon_{f}(n+N) / N} \sum_{u=0}^{U-1} h_{u} x\left(n+N-\alpha_{u}\right)+w(n) \\
=e^{j 2 \pi \varepsilon_{f} n / N} e^{j 2 \pi \varepsilon_{f}} \sum_{u=0}^{U-1} h_{u} x\left(n+N-\alpha_{u}\right)+w(n) \\
=e^{j 2 \pi \varepsilon_{f} n / N} e^{j 2 \pi \varepsilon_{f}} \sum_{u=0}^{U-1} h_{u} x\left(n-\alpha_{u}\right)+w(n),
\end{aligned}
$$

where $U$ denotes the multi-path number, $h_{u}$ represents the fading channel impulse response of $u$ path, $\alpha_{u}$ represents channel delay of $u$ path, and $w(n)$ represents Gaussian

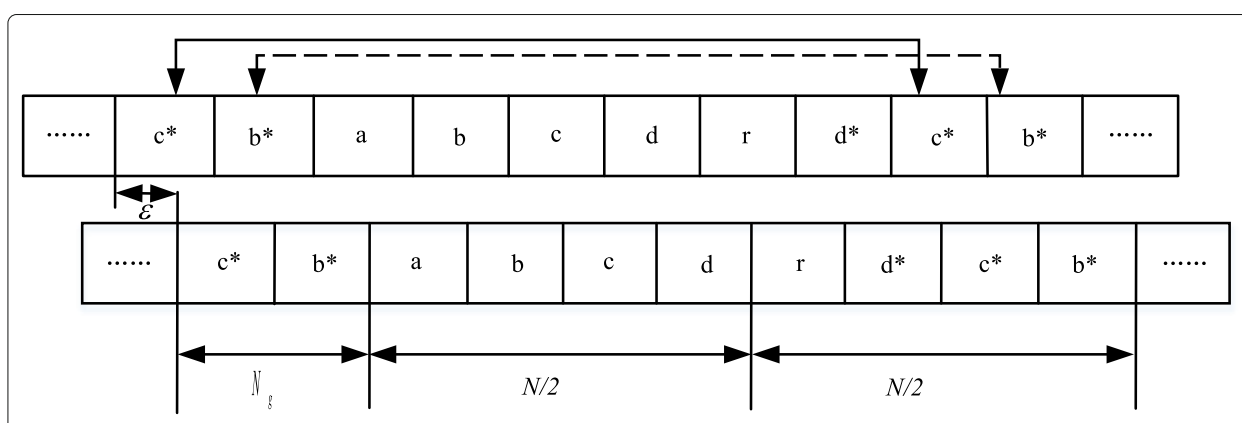

Fig. 6 Calculation process of FFO estimation 
noise. Then, the phase difference between the CP and the duplicate part of the OFDM symbol can be confirmed from Eq. (28) and (29) as follows.

$$
\phi=2 \pi \varepsilon_{f} .
$$

The phase estimation can be calculated through the received data as follows.

$$
\hat{\phi}=\operatorname{angle}(P(\hat{s}))=\operatorname{angle} \sum_{k=1}^{N_{g}} r^{*}(\hat{s}-k) r(\hat{s}+N-k) .
$$

Then, $\hat{\varepsilon}_{f}$ is figured out as:

$$
\hat{\varepsilon}_{f}=\hat{\phi} / 2 \pi=\left[\operatorname{angle}\left(\sum_{k=1}^{N_{g}} r^{*}(\hat{s}-k) r(\hat{s}+N-k)\right)\right] / 2 \pi .
$$

If $|\varepsilon|>1$, it is necessary to estimate $\varepsilon_{I}$. According to the analysis in Section 3, the influence of $\varepsilon_{I}$ is just the cyclical shift of the frequency domain data. As shown in Fig. 7, the position of the maximum correlation value can be achieved by cyclic correlation operation between the frequency domain signal of the received data and that of the original data.

Supposing $y_{k}, k=0,1, \ldots, N-1$ is the received signal after the compensation of FFO, $x_{k}, k=0,1, \ldots, N-1$ is the corresponding transmitted source data. Then, the IFO can be approximate calculated by:

$$
\varepsilon_{I}=\underset{s \in S}{\arg \max } \sum_{v=1}^{V}\left(x_{v} y_{s+v}^{*}\right),
$$

where $V$ is the estimation range of $\varepsilon_{I}$, that is, the length of the sliding window. $s$ is the window movement value, $s \in S, S=0,1, \ldots, N-1$.

\section{Results and discussion}

In this section, the bit error rate (BER) performance, timing metric curve and frequency offset mean square error (MSE) of F-OFDM and OFDM systems are compared by matlab

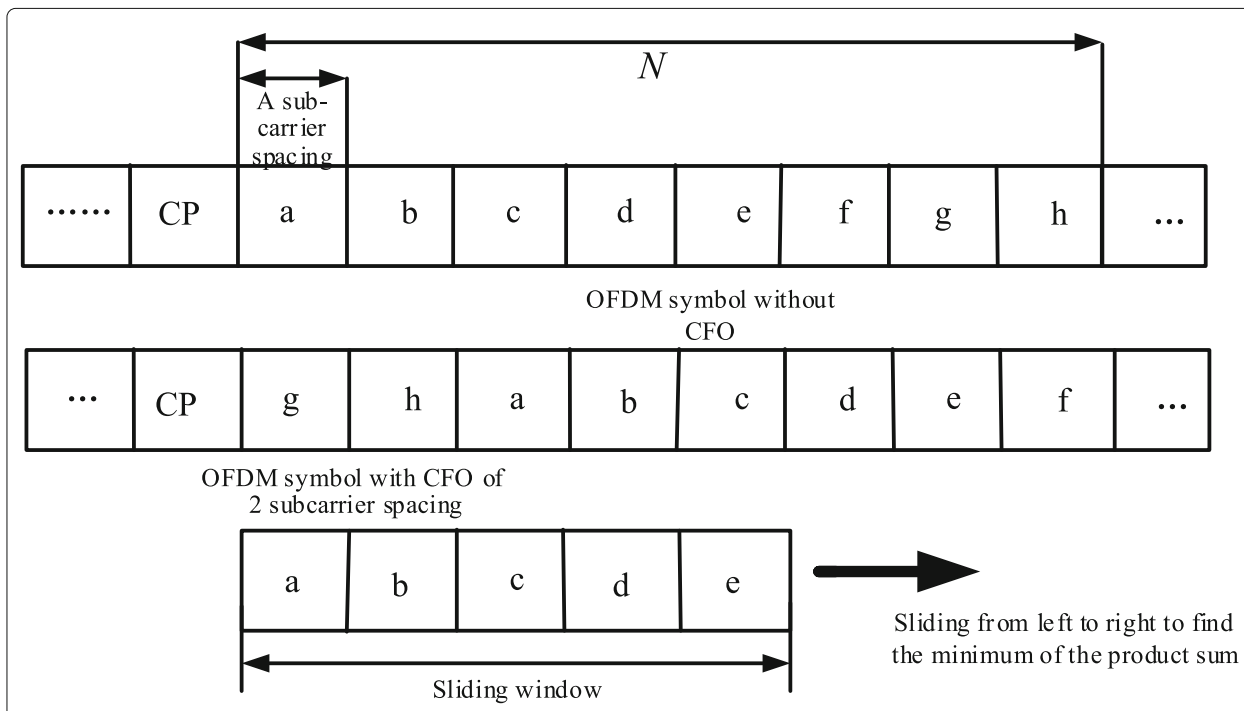

Fig. 7 Ca1lculation process of IFO estimation 
simulations. The simulation parameters of F-OFDM and OFDM systems are presented in Table 1, which use Monte Carlo simulation with $10^{5}$ cycles times.

Figure 8 simulates the BER curves of OFDM and F-OFDM system in Gauss channel (Fig. 8a) and Rayleigh fading channel (Fig. 8b), respectively. From Fig. 8a, it can be seen that the BER curves of F-OFDM and OFDM coincide in Gauss channel, that is, the two systems have the same performance, but F-OFDM has the specific advantages on flexible configuration parameters. Figure $8 \mathrm{~b}$ shows that the BER curves of subband 1 and subband 2 are gradually separated in Rayleigh fading channel, and the BER performance of subband 2 is better. This is because the subcarriers spacing of subband 2 is twice that of subband 1 , which makes the influence of doppler frequency shift on subband 2 less than that on subband 1 . However, whether subband 1 or subband 2, the BER curves of the two systems still coincide under the same parameter configuration. This results illuminate that the F-OFDM system can achieve flexible parameter configuration to meet the demand of multi-service in $5 \mathrm{G}$, while the system performance does not decline.

The comparison of the timing metric curves of different STO estimators in OFDM and F-OFDM systems is shown in Fig. 9, which simulates with SNR = 10dB in Rayleigh channel. Figure 9a, c, e, g correspond to the OFDM system, it is obvious that the curve of SC algorithm has a platform with a width of CP, while Minn's algorithm can reduce platform, but the main peak is not sharp, and there are several side peaks with significant amplitudes. Although the actual Park algorithm solves the above problems, it still could not completely eliminate the side peak interference. Liang Xiao's algorithm not only has the pulse-like timing metric curve, but also eliminates the side peaks completely. However, the spectral efficiency is low because three symbols are used for estimation. In contrast, FS-Park algorithm has a pulse timing metric curve similar to Liang Xiao's algorithm. What is more, only one symbol is enough to complete estimation, which significantly improves the spectrum utilization. Figure $9 \mathrm{~b}, \mathrm{~d}, \mathrm{f}, \mathrm{h}$ correspond to the F-OFDM system. Compared with the OFDM system, SC and Minn algorithms are most affected by system filters in F-OFDM system, followed by Park algorithm, FS-Park algorithm, and Liang Xiao's algorithm. The major reason is that the existence of filters has less impact on STO estimation by using the correlation of multiple symbols than by using the correlation of one symbol. However, the proposed algorithm has certain advantages in terms of some IOT systems with low timing and high spectral efficiency requirements.

Table 1 Basic parameters of system simulation

\begin{tabular}{lll}
\hline System model & F-OFDM/OFDM & \\
Parameter & Subband 1 & Subband 2 \\
Sampling rate & $30.72 \mathrm{MHz}$ & $30.72 \mathrm{MHz}$ \\
Bandwidth & $720 \mathrm{MHz}$ & $720 \mathrm{MHz}$ \\
Subcarrier spacing & $15 \mathrm{kHz}$ & $30 \mathrm{kHz}$ \\
Symbol period & $66.67 \mathrm{us}$ & $33.33 \mathrm{us}$ \\
FFT size & 2048 & 1024 \\
Number of protection spacing & 2 & 2 \\
Wave filter & Window function (Hanning window) \\
Modulation mode & QPSK & \\
Channel model & Gauss Channel/Rayleigh Fading Channel \\
Noise & White Gaussian noise \\
\hline
\end{tabular}



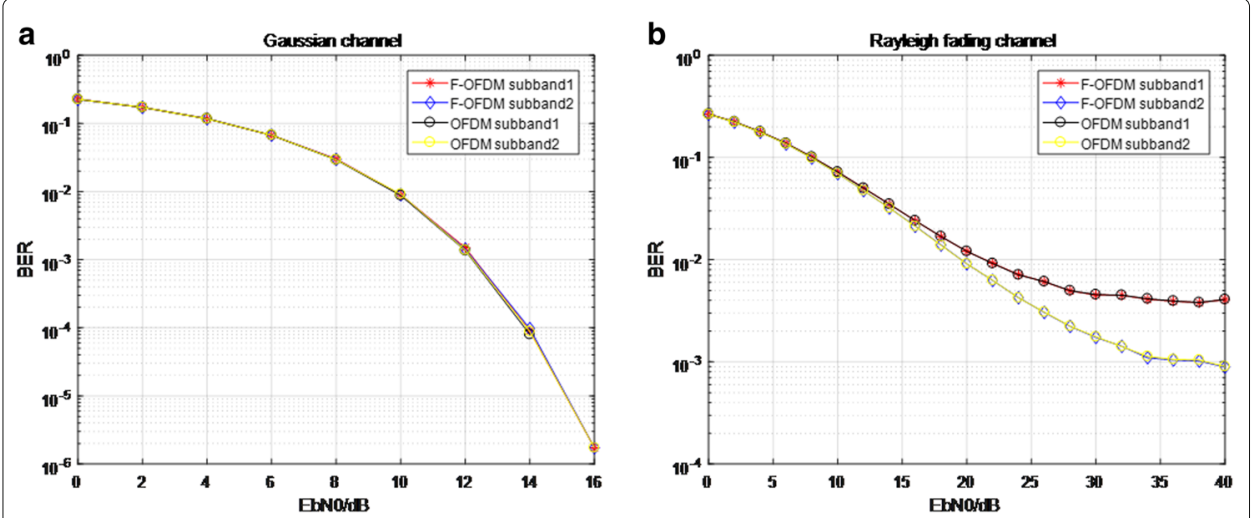

Fig. 8 BER comparison of OFDM and F-OFDM systems

Figure 10 shows the MSE curves of the different STO estimators, which simulates in F-OFDM system. It can be seen that the estimation performance of the Liang Xiao's algorithm is the best, followed by the FS-Park algorithm, and the estimation performance of the Park algorithm is the worst, which is also consistent with the results in Fig. 9.

Figure 11 shows the MSE curves of the different CFO estimators at $\varepsilon$ is $0.1,0.2,1.2$ respectively. When $\varepsilon$ is 0.1 , the CFO estimation performance of Liang Xiao's algorithm is the best, followed by FS-Park algorithm, and Park algorithm is the worst. This is because Liang Xiao's algorithm uses the correlation of two symbols to complete FFO estimation, so the estimation performance is optimal at the cost of increasing complexity. Both FS-Park algorithm and Park algorithm use one symbol to estimate CFO, but the STO estimation performance of FS-Park algorithm is better than Park algorithm, which makes it is less affected by timing estimation error when completing CFO estimation. When $\varepsilon$ is 0.2 , the performance of Liang Xiao's method declines sharply because the estimation range of FFO is only $(-0.125,0.125)$. When the absolute value of CFO is in the range of $(0.125$, $0.5)$, the accurate estimation can not be completed, while Park algorithm and FS-Park algorithm have no such limitation. However, when $\varepsilon$ is 1.2, only FS-Park algorithm has great estimation performance. The major reason is that Park algorithm can only estimate FFO, while Liang Xiao's method is unable to complete the accurate estimation of FFO, thus affecting the subsequent IFO estimation.

Figure 12 shows the estimation effects of the classical Park algorithm, Liang Xiao's method, and the FS-Park algorithm on different CFO values when SNR $=10$. It is clearly observed that when $\varepsilon$ is in the range of $(0.125,0.875)$ and $(1.125,1.4)$, the estimation performance of Liang Xiao's method deteriorates seriously. When $\varepsilon$ exceeds 1 , the estimation performance of classical Park algorithm declines sharply, while FS-Park algorithm still maintains great estimation performance.

\section{Conclusion}

This paper mainly studies the synchronization problem of F-OFDM system, which includes three parts. The first part studies and analyzes the influence of STO and CFO on F-OFDM system. It is found that the STO will lead to ISI between subbands, which indicates that synchronization is very necessary for F-OFDM system. The second part studies and points out the drawbacks of the classical Park algorithm, which makes it impossible 
a

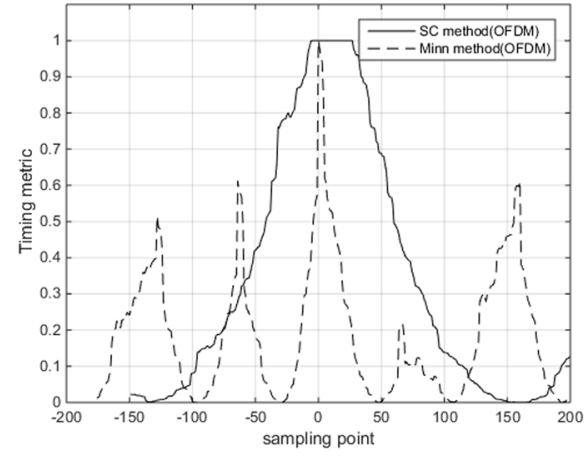

C

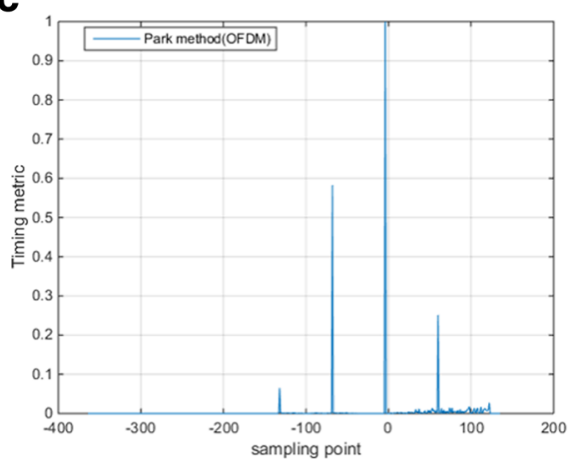

e

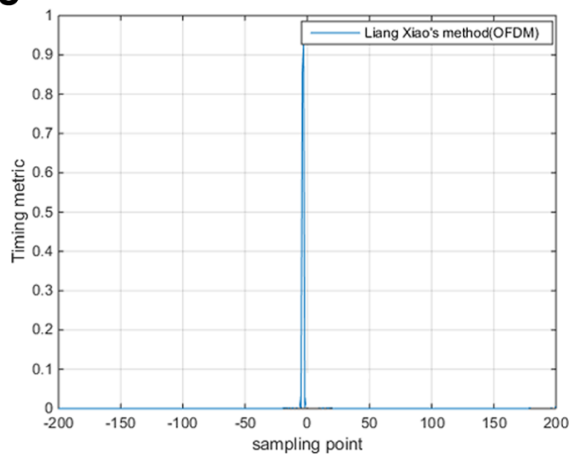

g

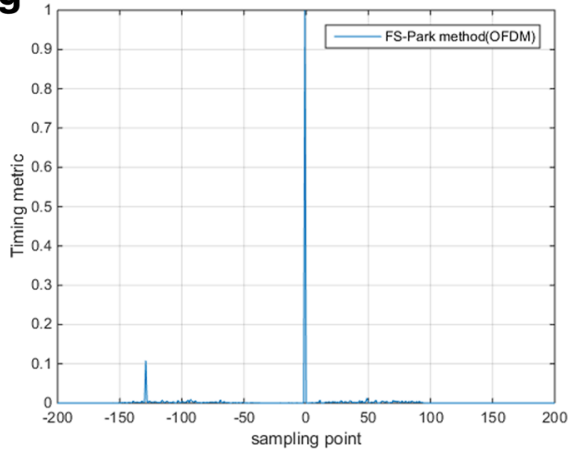

b

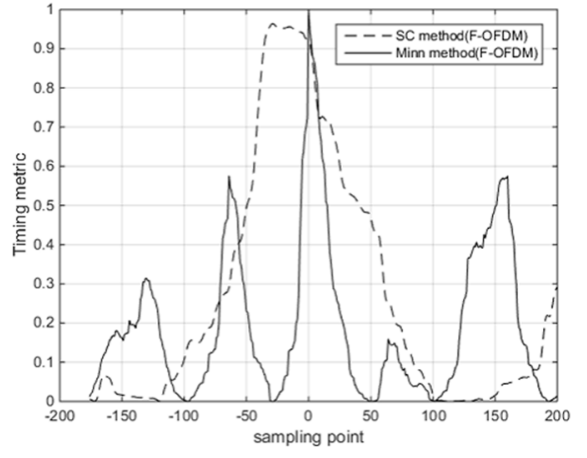

d

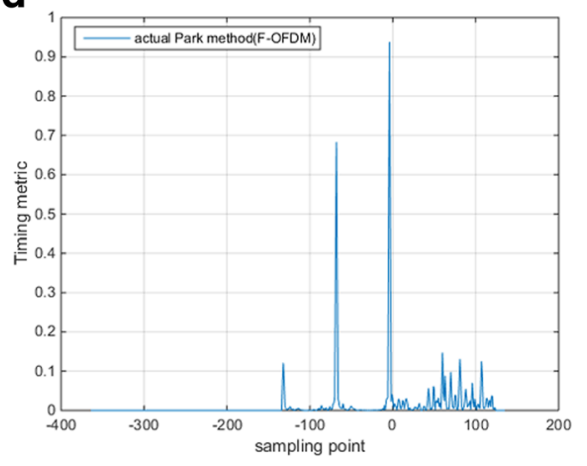

f

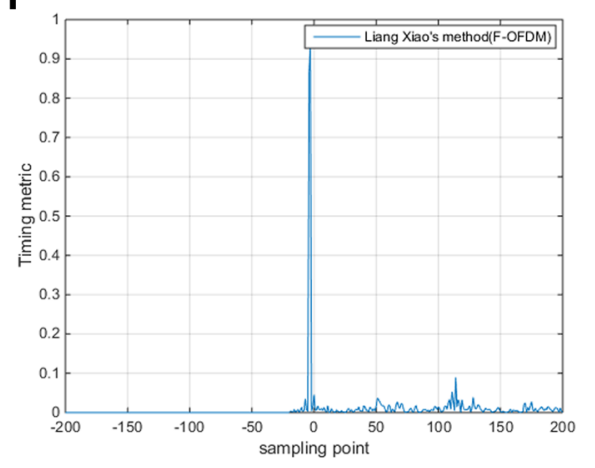

h

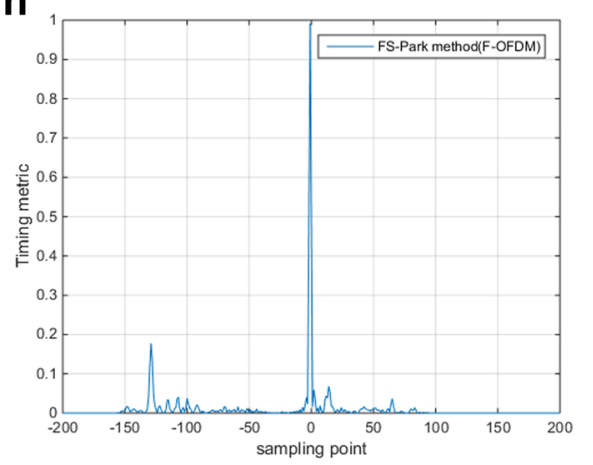

Fig. 9 Comparison of timing metric curves 


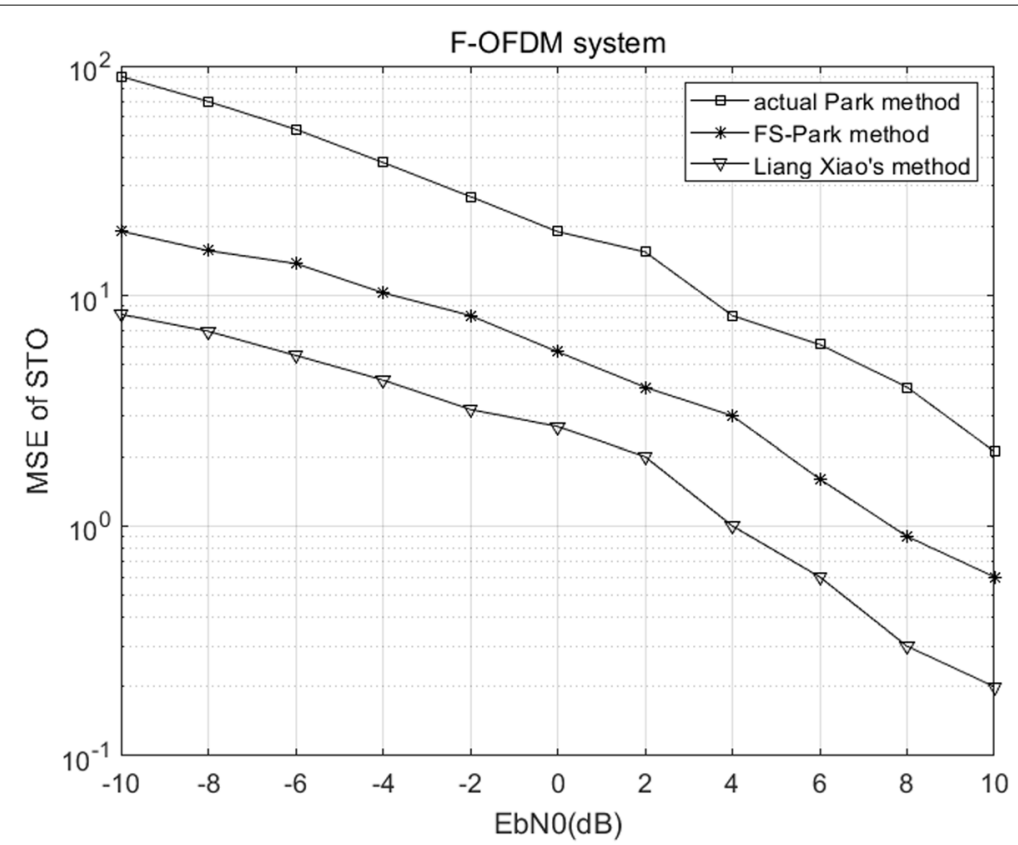

Fig. 10 MSE performance of various estimators with different STO

to implement. The third part proposes a synchronous algorithm, which is called FS-Park algorithm. It not only solves the problems that the timing metric curve of actual Park algorithm has many side peaks and the CFO estimation range of algorithm is only one subcarrier spacing, but also has higher spectral efficiency and more accurate CFO estimation than Liang Xiao's method. The core idea of these contributions is to design a new

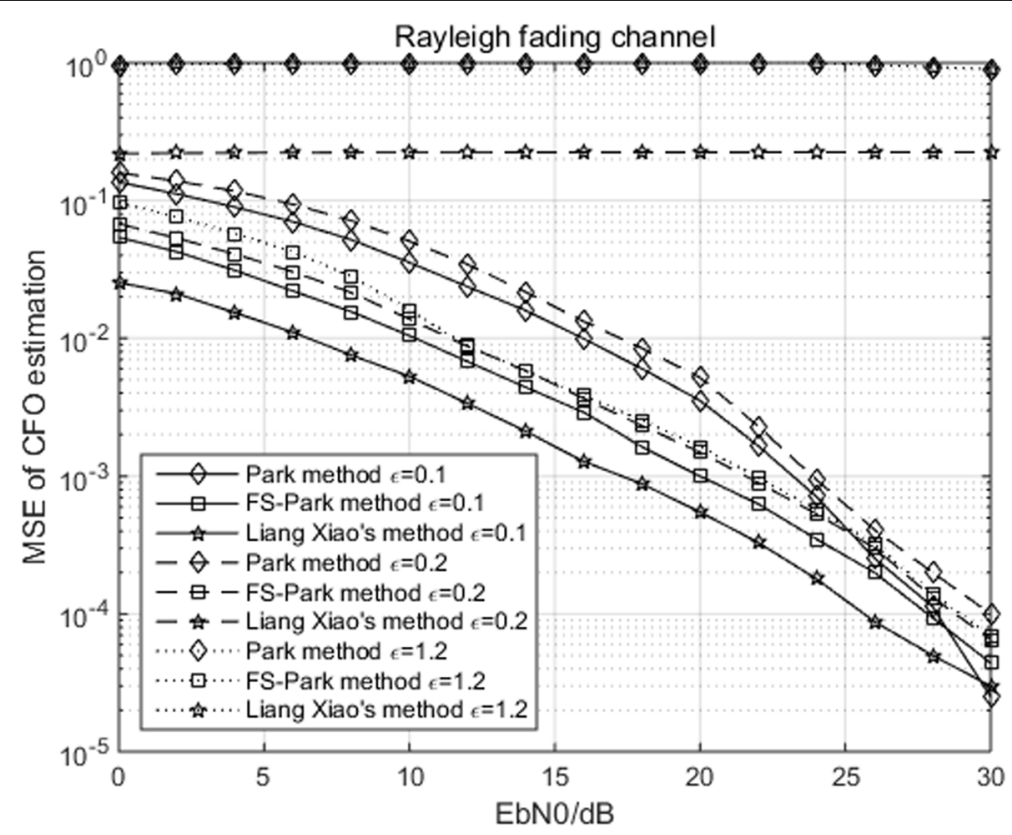

Fig. 11 MSE performance of various estimators with different CFO 


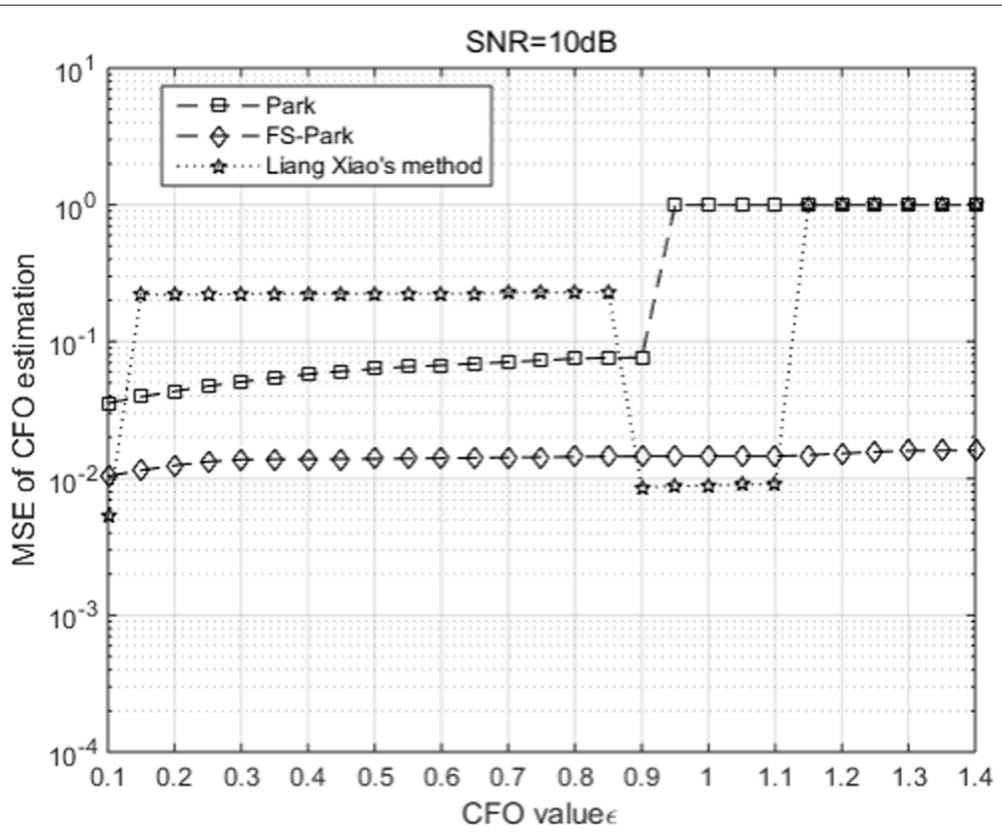

Fig. 12 MSE curves with different CFO

training sequence, which transmits real PN sequence in all subcarriers, then the corresponding time domain training symbol has a conjugate symmetric structure, and STO and CFO estimation can be achieved simultaneously with only one training symbol. The simulation results illustrate that the proposed algorithm will not only effectively eliminate the platform which causes the timing imprecision of the SC algorithm, but also can solve the problem that the peak value of the Minn algorithm is not sharp. Moreover, it can eliminate the large side peaks produced by SC, Minn, and Park algorithms radically, which has the same single pulse timing metric curve as Liang Xiao's method. In addition, compared with the classical Park algorithm and Liang Xiao's method, the proposed FS-Park algorithm is more suitable for timing and frequency synchronization of F-OFDM system because it has better estimation performance in the whole symbol range.

\section{Abbreviations}

4G: The fourth generation; 5G: The fifth generation; AWGN: Additive white Gaussian noise; CFO: Carrier frequency offset: CP: Cyclic prefix; FFO: Fractional frequency offset; FFT: Fast Fourier transform; F-OFDM: Filtered orthogonal frequency division multiplexing; FS-Park: Park frequency domain training sequence; ICl: Intercarrier interference; IFO: Integer frequency offset; IFFT: Inverse fast Fourier transform; IOT: Internet of things; ISI: Intersymbol interference; ML: Maximum likelihood; PN: Pseudorandom number; STO: Sample timing offset

\section{Acknowledgements}

Not applicable.

\section{Author's contributions}

XF and LC jointly proposed the FS-park synchronization algorithm and developed the mathematical derivation. MW applied the algorithm to the F-OFDM system and completed the simulation work of each part. The paper was written by $\mathrm{LC}$ and $\mathrm{MW}$ and revised by XF, MW, WZ, and KH. All authors read and approved the final manuscript.

Funding

The research in this article was supported by the National Natural Science Foundation of China (No. 61871327) and Shaanxi local standard project (SDBXM67-2020). 
Ethics approval and consent to participate

Not applicable

\section{Consent for publication}

The manuscript does not contain any individual person's data in any form (including individual details, images, or videos), and therefore, the consent to publish is not applicable to this article.

\section{Competing interests}

The authors declare that they have no competing interests.

\section{Author details}

${ }^{1}$ Chang'an University, 710064 Xi'an, China. ${ }^{2}$ Jushri Technologier Inc, 200050 Shanghai, China. ${ }^{3}$ Lawrence Technological University, 48075 Michigan, USA.

Received: 22 March 2020 Accepted: 10 August 2020

Published online: 01 September 2020

\section{References}

1. G. Li, T. Y. Li, M. K. Xu, et al., Sparse massive MIMO-OFDM channel estimation based on compressed sensing over frequency offset environment. EURASIP J. Adv. Signal Process. 2019(1), 1-13 (2019)

2. J. Abdoli, M. Jia, J. L. Ma, in 16th International Workshop on Signal Processing Advances in Wireless Communications, Filtered OFDM: a new waveform for future wireless systems (IEEE Signal Processing Society, Stockholm, 2015), pp. 66-70

3. L. Zhang, I. Ayesha, X. Pei, et al., Filtered OFDM systems, algorithms, and performance analysis for $5 \mathrm{G}$ and beyond. IEEE Trans. Commun. 66(3), 1205-1218 (2018)

4. S. Sharma, K. Thakur, in Proc. of the 2nd International Conference on Inventive Systems and Control, Carrier frequency offset in OFDM systems, (2018), pp. 369-373. https://doi.org/10.1109/ICISC.2018.8399096

5. R. Fan, Research and implementation of FBMC synchronization algorithm. ((University of Electronic Science and Technology of China, Chendu, 2016)

6. X. J. Wang, T. Wild, F. Schaich, in 81st Vehicular Technology Conference, Filter optimization for carrier frequency and timing offset in universal filtered multi-carrier systems (IEEE, Glasgow, 2015)

7. X. Q. Fu, Research on frequency offset estimation algorithm for filtered-OFDM. (Southwest Jiaotong university, Chengdu, 2017)

8. J. J. V. D. Beek, M. Sandell, P. O. Borjesson, ML estimation of time and frequency offset in OFDM systems. IEEE Trans. Signal Process. 45(7), 1800-1805 (1997)

9. D. Lanstrom, S. K. Wilson, Symbol time offset estimation in coherent OFDM systems. IEEE Trans. Commun. 50(4), 545-549 (2002)

10. Q. Li, H. W. Li, B. Cai, et al., Approach to frequency offset estimation of OFDM based on PN sequences distributed null subcarriers. J. Signal Process. 29(1), 60-67 (2013)

11. T. M. Schmidl, D. C. Cox, Robust frequency and timing synchronization for OFDM. IEEE Trans. Commun. 45(12), 1613-1621 (1997)

12. H. Mimm, M. Zeng, V. K. Bhargava, On timing offset estimation for OFDM systems. IEEE Commun. Lett. 4(7), 242-244 (2000)

13. B. Park, H. Cheon, C. Kang, et al., A novel timing estimation method for OFDM systems. IEEE Commun. Lett. 7(5), 269-272 (2003)

14. X. Liang, Research on synchronization and receiver of new broadband multi-carrier systems. (University of Electronic Science and Technology, Chengdu, 2018)

15. Y. L. Gu, Research on frequency offset estimation algorithm in FBMC and OFDM system. (Beijing University of Posts and Telecommunications, Beijing, 2018)

\section{Publisher's Note}

Springer Nature remains neutral with regard to jurisdictional claims in published maps and institutional affiliations. 